\title{
Diagnóstico de la competencia comunicativa en inglés de un grupo de escolares chilenos: puntos de encuentro con su perfil estratégico
}

\author{
A diagnosis of the English communicative competence \\ of a group of Chilean students - Some common issues \\ with their strategic profile
}

María Gabriela Sanhueza Jara ${ }^{1}$ Gina América Burdiles Fernández ${ }^{2}$

Resumen

Recientemente, el Ministerio de Educación de Chile aplicó por primera vez una evaluación de las habilidades receptivas en inglés a todos los estudiantes de tercer año de enseñanza media del país. Este diagnóstico permitió advertir que en las áreas de comprensión auditiva y comprensión lectora alcanzan niveles insuficientes. Sin embargo, aún es muy poco lo que se ha investigado, en el contexto educacional chileno, acerca del estado de la competencia comunicativa de nuestros jóvenes en lengua inglesa. La presente investigación se propone establecer algunos puntos de encuentro entre la descripción de la competencia comunicativa en inglés de un grupo de escolares chilenos y el perfil de estrategias de aprendizaje de lengua extranjera que les caracteriza. Este estudio corresponde a uno descriptivo, transversal, de caso único. Los datos analizados provienen de los informes de resultados de la aplicación del examen First Certificate in English y de un perfil estratégico de aprendizaje de inglés como idioma extranjero obtenido a partir del cuestionario Strategy Inventory for Language Learning.

Palabras clave

Aprendizaje de inglés, competencia comunicativa, estrategias de aprendizaje de lengua extranjera.

Abstract

Recently, the Chilean Ministry of Education has applied for the first time, an assessment of the receptive skills in the English language of every Chilean third year high school student. This diagnosis evinced that in the areas of listening and reading comprehension, the students' level was unsatisfactory. Nevertheless, what has been investigated with respect to the Chilean school students' level of communicative competence in English has been very little. The present investigation seeks to establish some common issues between the description of the communicative competence of the Chilean students and their typical, foreign language learning strategic profile. This unique case study is one that is descriptive and transversal. Analyzed data was gathered from the First Certific ate in English examination reports and a strategic learning profile of English as a foreign language which was obtained from the questionnaire Strategy Inventory for Language Learning.

Key words

English language learning, communicative competence, foreign language learning strategies.

Artículo recibido el 8 de junio de 2011 y aprobado el 19 de julio de 2012

1 Universidad Católica de la Santísima Concepción, Concepción, Chile. Correo electrónico: gabrielasanhueza@ucsc.cl

2 Universidad Católica de la Santísima Concepción, Concepción, Chile. Correo electrónico: gburdiles@ucsc.cl 
Los distintos enfoques de enseñanza del inglés han evolucionado en forma paralela al concepto de competencia comunicativa y a las diversas propuestas acerca de sus componentes (Hymes, 1972; Widdowson, 1978; Canale y Swain, 1980; Canale, 1983; Van Ek, 1984; Medina, 1998; Oliveras, 2000; Pulido y Pérez, 2004). En un sentido amplio, se entiende por competencia comunicativa a las capacidades, conocimientos y habilidades lingüísticas y paralingüísticas que regulan el sentido de las interacciones comunicativas en contextos socioculturales específicos (Medina, 1998; Pulido y Pérez, 2004).

La competencia comunicativa en idioma extranjero ha cobrado gran importancia en el contexto de las nuevas tendencias socioeconómicas, los nuevos perfiles de empleos, las nuevas relaciones interculturales y la aceleración vertiginosa de las innovaciones que plantea la sociedad globalizada en que vivimos. No ajenas a este fenómeno, las políticas educativas chilenas han orientado la enseñanza-aprendizaje del inglés hacia el logro de niveles de competencia específicos para las distintas etapas de la educación formal. Estos niveles de dominio guardan correspondencia con los parámetros internacionales de estandarización de competencia comunicativa en idioma extranjero, establecidos por el Consejo de Europa a través de la Asociación Europea de Examinadores de Idiomas (ALTE). Dicha organización ha implementado evaluaciones tendientes a comprobar distintos niveles de competencia comunicativa en inglés, una de las cuales es el First Certificate in English (FCE).

A fines del año 2010, el Ministerio de Educación de Chile evaluó, por primera vez, el nivel de logro de los objetivos fundamentales y contenidos mínimos obligatorios correspondientes al subsector idioma extranjero inglés del Marco Curricular, por parte de los estudiantes de tercer año de educación media, solo en las áreas de comprensión auditiva y comprensión lectora. Al respecto, cabe hacer notar que esta es una medición parcial del nivel de competencia comunicativa que el Ministerio se propuso desarrollar al establecer el nivel B1 del Marco Común Europeo como meta para estudiantes que egresan de cuarto año de educación media en
Chile. Para este caso, los resultados informan que uno de cada diez alumnos alcanzó un nivel considerado "básico" y solo el 11\% de los alumnos de 3 º medio comprende frases cotidianas y textos breves y sencillos, situación que evidencia que los esfuerzos realizados por el Ministerio de Educación en cuanto a la enseñanza y aprendizaje de inglés en el nivel escolar chileno son todavía infructuosos.

En Chile son escasos los estudios sobre competencia comunicativa en inglés como lengua extranjera. Doddis, Novoa y Tabilo (2003) investigaron el uso de estrategias léxicas en la habilidad de producción oral, en estudiantes universitarios de distintos niveles de desarrollo de competencia comunicativa. Otro estudio que apunta al mismo nivel educativo es el de Cartes, Solar y Quintana (2004), quienes, de manera experimental, demuestran la positiva incidencia de una metodología de aula inteligente en el aprendizaje de inglés. No hay investigaciones sobre estudiantes de enseñanza media, a excepción de la reflexión de Rosas (1997), desde una perspectiva cognitiva, sobre las posibles causas de la falta de competencia de los alumnos egresados de enseñanza media en la asignatura de inglés como lengua extranjera, con respecto a la comprensión y producción oral y escrita. En relación con estudiantes de enseñanza básica, destaca el estudio de Germany y Cartes (2000), quienes desarrollaron un estudio transaccional correlacional, en el que describieron el nivel lingüístico del léxico disponible de escolares chilenos que estudian inglés como lengua extranjera en la enseñanza formal, en distintos tipos de establecimientos educacionales de la Octava Región. Las autoras concluyeron que la variable disponibilidad léxica presenta un incremento significativo dependiendo del establecimiento educacional en que esta haya sido medida y observada. Como es posible observar, en el contexto local, el estado de las competencias comunicativas de escolares chilenos es un campo inexplorado.

La finalidad del presente estudio es establecer algunos puntos de encuentro entre la descripción de la competencia comunicativa en inglés de un grupo de escolares chilenos y el perfil de estrategias de aprendizaje de lengua extranjera que los carac- 
teriza. En consecuencia, nos hemos propuesto, por una parte, describir detalladamente el nivel de competencia comunicativa en inglés alcanzado por un grupo de escolares chilenos en el examen FCE y, por otra, establecer asociaciones entre estos niveles de competencia obtenidos en los diversos aspectos del FCE y el perfil estratégico que caracteriza a este grupo de estudiantes. Los datos con los que trabajamos provienen de los informes de resultados de la aplicación del FCE y de un perfil estratégico de aprendizaje de inglés como idioma extranjero obtenido a partir del Strategy Inventory for Language Learning (SILL).

Dado que esta investigación constituye una primera aproximación al tema desde la realidad de un colegio particular bilingüe chileno, los resultados informan de una situación que hasta ahora no ha sido diagnosticada en el país. El estudio que se describirá en las páginas siguientes considera que el diagnóstico de la realidad nacional, respecto de la competencia comunicativa en inglés de alumnos chilenos, es un importante paso para diseñar, implementar y evaluar intervenciones tendientes a optimizar los procesos de enseñanza-aprendizaje del inglés como idioma extranjero en los colegios.

\section{Marco teórico}

\section{El enfoque comunicativo en la enseñanza de lenguas extranjeras}

La enseñanza de lenguas se basó, hasta la década de los 70, en un enfoque estructural o gramatical, que se centraba en los aspectos sistemáticos de la lengua. Luego, surge un enfoque comunicativo en la enseñanza de lenguas, inspirado en la distinción que Chomsky (1965) hizo entre "competencia" y “actuación”. Según este autor, la competencia se relaciona con el conocimiento inconsciente de reglas lingüísticas, las que se construyen a partir de una gramática universal básica e innata, de carácter hipotético; la actuación, en tanto, se relaciona con el uso concreto de la lengua.

Este enfoque comunicativo en la enseñanza de lenguas extranjeras, se convierte en el marco para modelos didácticos destinados a capacitar a los estudiantes para comunicarse en contextos reales, haciendo uso funcional de la lengua. A partir de él, se subraya el potencial funcional y comunicativo de la lengua (Halliday, 1970); por tanto, la atención se traslada de "lo que es" el lenguaje a lo que "se hace" con el lenguaje. Esta mirada subraya la noción de uso que debe primar en el aula, de modo que los alumnos se apropien del idioma y sean competentes al momento de usarlo espontáneamente en contextos de la vida diaria.

Así, basado en sus estudios acerca de la relación entre los sistemas lingüísticos y su dimensión comunicativa en el texto y en el discurso, Widdowson (1978) establece que no basta con conocer una gama de estructuras lingüísticas y saber usarlas, sino que se requiere producir enunciados significativos que respondan a la intención de aquellos producidos por un interlocutor. En síntesis, el enfoque comunicativo en la enseñanza de la lengua asume que la lengua es comunicación y el objetivo de la enseñanza de la lengua ha de ser desarrollar lo que Hymes llamó la competencia comunicativa.

Como consecuencia de estos nuevos conceptos que se manifiestan en cambios en la enseñanza de lenguas extranjeras, surgen los programas de lengua denominados métodos nocional-funcionales, como antesala del enfoque comunicativo propiamente tal. En dichos programas se relacionan los contenidos semánticos (nociones) con lo que se puede hacer con la lengua (funciones). De este modo, enseñar una lengua desde esta perspectiva tiene como propósito fundamental que el alumno la utilice con la posibilidad de adquirir conocimientos a través de ella, de expresar y comprender ideas, de interactuar con el medio y apropiarse de la cultura extranjera como también valorar la propia.

Los programas de inglés orientados por el enfoque comunicativo deben considerar al alumno como un activo participante en la interpretación, expresión y negociación de significados. Es a él a quien deben dársele las mayores y mejores oportunidades para interactuar con el idioma que está aprendiendo, intentando crear situaciones reales que demanden la necesidad de comunicación auténtica. A diferencia del método audio lingual que privilegiaba el discurso 
oral y la secuenciación del desarrollo de habilidades, este enfoque integra todos los aspectos de la lengua desde los inicios de la enseñanza y no sigue un orden fijo en el desarrollo de estos; así concurren indistintamente en la sala de clases de lengua la comprensión oral, la producción oral, la comprensión escrita y la producción escrita. Además, se da importancia al uso de materiales auténticos que contribuyan al conocimiento cultural y a proveer de significación al ambiente de aprendizaje.

En consecuencia, el rol del profesor es el de facilitador de la comunicación, de creador de las condiciones ambientales que estimulen la motivación en el proceso de enseñanza-aprendizaje y ofrezcan un input significativo que genere en los aprendices la necesidad de comunicarse.

\section{Competencia comunicativa}

El concepto de competencia comunicativa es inherente al enfoque comunicativo, pues el desarrollo de esta constituye el propósito fundamental al momento de enseñar y aprender lenguas extranjeras, bajo este enfoque. De tal forma, el concepto de competencia comunicativa ha evolucionado en forma paralela al asentamiento de las bases del enfoque comunicativo.

El concepto de competencia comunicativa (Hymes, 1972) surge a partir de la diferenciación que establece Chomsky (1965) entre competencia y actuación, en cuanto considera que el aprender una lengua no se circunscribe a la mera competencia lingüística, pues al usar la lengua también, y sobre todo, se conjugan factores no cognitivos que están determinados por el contexto situacional donde ocurren los actos de habla.

Otros autores (Canale y Swain, 1980; Van Ek, 1984; Lomas y Osoro, 1994; Medina, 1998; Oliveras, 2000; Pulido y Pérez, 2004) han ampliado el concepto de competencia comunicativa, en cuanto reconocen que en la comunicación no solo intervienen aspectos cognitivos sino que también cobran importancia otros aspectos tales como factores lingüísticos, sociolingüísticos, discursivos, estratégicos, psicológicos, afectivos, comportamentales, socioculturales e interculturales.
En esta investigación se asume el concepto de competencia comunicativa como el sistema configuracional (Medina, 1998) que involucra el desarrollo de todas las competencias antes descritas: competencia gramatical (o lingüística), competencia discursiva, competencia sociolingüística, competencia estratégica, competencia sociocultural, competencia de aprendizaje, competencia cognitiva, competencia afectiva, competencia comportamental y competencia intercultural.

\section{Evaluación de la competencia comunicativa en lengua extranjera: examen First Certificate in English (FCE)}

Desde 1991, como resultado de los crecientes flujos de trabajadores de unos países europeos a otros, se ha hecho necesario unificar criterios respecto a los diversos niveles de dominio de idiomas extranjeros, con el fin de poder homologar calificaciones obtenidas en diferentes países en relación a criterios comunes. El Consejo de Europa con la cooperación de ALTE, un organismo perteneciente a la Universidad de Cambridge que facilita exámenes y certificaciones para estudiantes de idiomas, ha dedicado sus esfuerzos a la redacción de criterios descriptivos que permitan la comparación de los diversos exámenes rendidos en distintos puntos de Europa. Como fruto de este trabajo conjunto surge la descripción de los contenidos para seis niveles (A1, A2, B1, B2, C1 y C2) que, en su conjunto, conforman el Marco común europeo de referencia para las lenguas: aprendizaje, enseñanza y evaluación (Instituto Cervantes, 2002). Este documento proporciona una base común para la elaboración de programas de lenguas, orientaciones curriculares, exámenes, manuales y materiales de enseñanza en Europa, contribuyendo de este modo a facilitar la movilidad entre los ámbitos educativo y profesional.

El examen FCE es uno de los exámenes diseñados y administrados por la Universidad de Cambridge y apunta a la acreditación del nivel B2 establecido por el Consejo de Europa y al nivel 3 de la estructura ALTE. Más de 270.000 hablantes de inglés como lengua extranjera rinden este examen en más de 100 países. 
El enfoque teórico desde el que se diseña el FCE responde al declarado por el Consejo de Europa; un enfoque orientado a la acción que considera a quienes aprenden una lengua como agentes sociales, es decir, como miembros de una sociedad que realizan tareas -no solo lingüísticas- en contextos específicos. Bajo este enfoque, el uso y aprendizaje de la lengua comprende el desarrollo de una serie de competencias, tanto generales como competencias comunicativas, necesarias para actuar socialmente (Instituto Cervantes, 2002). En concordancia con lo establecido por el Marco común europeo, el FCE evalúa tanto las competencias generales, entendidas como la suma de conocimientos, destrezas y características individuales que permiten a una persona realizar acciones, como las competencias comunicativas, es decir, las que posibilitan a una persona actuar utilizando específicamente medios lingüísticos (Instituto Cervantes, 2002).

El FCE se establece como la acreditación de un nivel intermedio superior de competencia comunicativa en lengua inglesa, el cual supone que el usuario de la lengua es capaz de usar las estructuras principales de la lengua con confianza, usar una amplia gama de vocabulario y un repertorio apropiado de estrategias comunicativas en una variedad de situaciones sociales. La comprensión demostrada, tanto de textos orales como escritos, debe superar la identificación de información basada en hechos y ser capaz de distinguir entre ideas generales y específicas. Se espera, asimismo, la capacidad de producir una amplia gama de textos escritos, demostrando habilidad tanto para describir y narrar acontecimientos como para desarrollar textos argumentativos. Además, se espera conciencia del registro y las convenciones de cortesía y grados de formalidad expresados a través del lenguaje. Por último, en este nivel el usuario debe poder hacer frente a situaciones inesperadas recurriendo a su habilidad y flexibilidad en el uso, no solo de conocimientos lingüísticos, sino también de las estrategias de comunicación que ha desarrollado durante el aprendizaje de la lengua.

Este examen es reconocido por instituciones académicas y empresas de todo el mundo, ya que garantiza un nivel de competencia comunicativa que le permite al usuario de la lengua desenvolverse de tal forma que pueda continuar sus estudios de inglés en un país donde este idioma sea lengua materna, o bien realizar trabajos en áreas donde se requiera el uso del idioma.

\section{Estrategias de aprendizaje y aprendizaje de lenguas extranjeras}

Aprender una lengua extranjera implica también diferencias en relación con el uso de estrategias de aprendizaje. A este respecto, Ellis (2003, p. 165) informa que hay evidencia empírica que respalda la idea de que los aprendices de una lengua extranjera, o segunda lengua, usan con mayor frecuencia que los hablantes nativos estrategias de aprendizaje. Oxford (1990, p. 6), agrega que si bien muchas estrategias de aprendizaje son igualmente usadas en el caso de aprendizaje de LM, L2 o LE, el uso de algunas estrategias puede resultar más fácil en contextos de lengua materna o segunda lengua que al aprender una lengua extranjera.

Las estrategias de aprendizaje han sido consideradas especialmente importantes para el aprendizaje del idioma, puesto que constituyen herramientas para el estudiante en el involucramiento activo y autodirección del aprendizaje, lo que es esencial para el desarrollo de la competencia comunicativa (Oxford, 1990; Richards y Lockhart, 1994; Monereo, 2004; Casar y Hernández, 2000).

Oxford (1990) las define como acciones específicas que un aprendiz ejecuta a fin de hacer del aprendizaje algo más rápido, entretenido, autodirigido y extrapolable a situaciones diferentes. En coincidencia con Richards y Lockhart (1994), Oxford (1990) sugiere que las estrategias para el aprendizaje de una lengua extranjera o segunda lengua se caracterizan por los siguientes rasgos: contribuir a la competencia comunicativa, permitir a los estudiantes ser más autodirigidos, expandir el rol de los profesores, orientarse a la resolución de problemas, ser acciones específicas asumidas por los aprendices, involucrar varios aspectos del aprendizaje (no solo el cognitivo), apoyar directa e indirectamente el aprendizaje, no ser siempre observables, generalmente, ser desarrolladas de forma consciente, ser susceptibles de enseñanza, ser flexibles e influenciadas por una variedad de factores. 


\section{Clasificación y evaluación de las estrategias de aprendizaje del inglés como lengua extranjera}

Existen diversos sistemas de clasificación de las estrategias de aprendizaje (Rubin, 1975; Dansereau et al., 1975; O'Malley et al, 1985; Wenden y Rubin, 1987; O'Malley y Chamot, 1987; Bialystok, 1981; Tarone, 1983; Sutter, 1989; Cohen, 1990; Oxford, 1990; Stern, 1992; Ellis, 1994). Una de las propuestas más relevantes por su protagonismo en los ámbitos investigativo, teórico y metodológico, es la de la investigadora Rebecca Oxford (1990), quien diseñó un sistema de clasificación no-taxonómico de estrategias de aprendizaje, de fácil comprensión, detallado y sistémico.

Oxford (1990) clasifica las estrategias de aprendizaje de idioma en dos grandes grupos: las estrategias directas, relacionadas específicamente con la lengua meta, y las estrategias indirectas, que tienen relación con el manejo global del aprendizaje. Cada uno de estos dos grupos se subdivide en tres subcategorías. Las estrategias directas las componen las estrategias de memorización, que se relacionan con el recuerdo y recuperación de información nueva; las estrategias cognitivas, para entender y producir la lengua, y las estrategias de compensación, aquellas tendientes a vencer los vacíos lingüísticos que se pudieran presentar al momento de usar la lengua. Las estrategias indirectas, por su parte, las conforman las estrategias metacognitivas, las cuales coordinan los procesos de aprendizaje; las estrategias afectivas, que regulan las emociones, y las estrategias sociales, que permiten el aprendizaje cooperativo.

Entre los rasgos característicos de las estrategias de aprendizaje de lenguas extranjeras está precisamente el que no siempre son observables (LessardClouston, 1997; Oxford, 1990; Richards y Lockhart, 1994), lo que dificulta la medición del uso de estrategias por parte de aprendices. Así las cosas, ha sido necesario recurrir a técnicas de evaluación de corte metacognitivo, entre las que destaca, no obstante sus limitaciones (Chamot, 2004), el cuestionario como método más frecuente y eficiente (Allwright, 1981; Cohen y Hosenfeld, 1981; Hosenfeld et al, 1981; Rubin, 1981; O'Malley et al, 1985; Wenden, 1986; Chamot et al, 1987; Mc Groarty, 1987).
Uno de los cuestionarios más utilizados en las dos últimas décadas para estudiar uso de estrategias en un gran número de lenguas extranjeras es el SILL, diseñado por Rebecca Oxford (1990) basándose en su propio sistema de estrategias de aprendizaje, anteriormente reseñado. Este instrumento es un cuestionario estructurado de autoinforme, cuya versión 7.0 está dirigida a hablantes de otros idiomas que aprenden inglés como segunda lengua o lengua extranjera.

Las preguntas de este cuestionario están organizadas en las seis categorías ya descritas, que conforman el sistema de estrategias de aprendizaje ideado por Oxford. Según Oxford (1996), a diferencia de muchos otros cuestionarios de estrategias que se han publicado (Bialystok, 1981; Politzer, 1983; Politzer y McGroarty, 1985; McGroarty, 1987; Chamot et al., 1987; Danserau, 1975), el SILL incluye factores sociales y afectivos, además de otros factores más intelectuales (cognitivos) y de manejo ejecutivo (metacognitivos).

El SILL es reconocido por su gran validez y confiabilidad (Oxford y Burry-Stock, 1995) y ha sido extensamente analizado y sometido a prueba en forma práctica, tanto en el aula como en investigaciones realizadas en distintas partes del mundo, con estudiantes de distintas lenguas maternas (Cohen et al, 1996; Nyikos y Oxford, 1993; Olivares-Cuhat, 2002; Oxford, 1990, 1996; Oxford y Burry-Stock, 1995; Wharton, 2000).

\section{Marco metodológico}

\section{El estudio}

La presente investigación corresponde a un estudio descriptivo, transversal; es de caso único, dado que considera una muestra de sujetos de un colegio chileno, bilingüe inglés-español, como unidad única de investigación.

\section{Objetivos}

1. Describir el nivel de competencia comunicativa en inglés alcanzado por un grupo de escolares chilenos en el examen FCE. 
2. Establecer relaciones entre los niveles de competencia comunicativa en inglés obtenidos por un grupo de escolares chilenos en el examen FCE y su perfil estratégico según el SILL.

\section{La muestra}

El universo corresponde a la totalidad de alumnos de colegios particulares bilingües inglés-español que rindieron en Chile el examen FCE, el año 2003. La muestra estuvo constituida por 15 estudiantes -5 hombres y 10 mujeres- del colegio seleccionado, que rindieron el FCE en esa ocasión; sus edades fluctuaban entre 15 y 17 años. Estos sujetos se caracterizaron por tener un rendimiento académico destacado (promedio de notas superior a 6,0). El $87 \%$ de ellos se integró a la institución escolar en cursos de la educación general básica, cuestión que significa un promedio de 3.000 horas pedagógicas de enseñanza-aprendizaje del inglés como idioma extranjero.

El colegio al cual pertenece la muestra es un colegio particular bilingüe inglés-español de Concepción, Chile, cuyos planes y programas contemplan la enseñanza del inglés como prioritaria desde $1^{\circ} \mathrm{a}$ $6^{\circ}$ básico. Es así que la mayoría de las asignaturas se dictan en esta lengua y desde $7^{\circ}$ básico a $4^{\circ}$ medio, la asignatura de inglés ocupa 6 horas pedagógicas a la semana. Como este establecimiento participó por primera vez de una evaluación externa internacional estandarizada, la muestra se caracteriza por haber sido el primer y único grupo de alumnos que había rendido el examen FCE.

\section{Instrumentos y técnicas de recolección de información}

La información recogida correspondió al nivel de competencia comunicativa en inglés como idioma extranjero, y se obtuvo de documentos oficiales que informan los resultados alcanzados por cada participante en la aplicación del examen FCE. Además, se trabajó con un perfil estratégico de aprendizaje de inglés como idioma extranjero, obtenido mediante la aplicación del SILL.

- Informe de resultados del examen FCE
La Universidad de Cambridge designa, en cada uno de los países en que aplica el FCE, centros autorizados que se encargan de su administración simultánea en diferentes partes del mundo. El examen, una vez contestado, es devuelto a Cambridge para su corrección. La parte oral es evaluada in situ por dos examinadores locales acreditados y los resultados son enviados a Cambridge para su inclusión en la evaluación global del mismo.

El FCE está constituido por cinco apartados (papers) que miden la comprensión lectora, expresión escrita, uso del idioma, comprensión auditiva y expresión oral. Los tres primeros apartados se evalúan en una primera sesión y los dos últimos en una segunda sesión desarrollada al día siguiente.

El primer apartado evalúa la habilidad para leer y comprender textos literarios y no literarios; en un plazo máximo de 75 minutos, se espera que el candidato demuestre la comprensión de ideas generales, ideas específicas, estructuras textuales y deduzca significados a través de cuatro tipos de ejercicios de comprensión lectora. El segundo apartado evalúa la habilidad del candidato en expresión escrita a través de la redacción de textos de una extensión de entre 120 y 180 palabras, cubriendo una gama de temas provenientes de textos literarios $\mathrm{u}$ otros seleccionados para el examen; esta sección consta de dos tipos de ejercicios que en su desarrollo no deben exceder los 90 minutos. El tercer apartado lo constituyen 5 tipos de ejercicios relativos al uso del idioma y el plazo para su desarrollo es de 75 minutos como máximo. En este apartado, se espera que el candidato demuestre su conocimiento y control del sistema de la lengua, completando diferentes tareas a nivel de texto y oración, mediante ejercicios como completación de información, parafraseo, formación y transformación de palabras e identificación de errores en textos dados. El cuarto apartado incluye cuatro tipos de ejercicios que deben realizarse dentro de 40 minutos; evalúa la habilidad para comprender el significado de enunciados orales a través de la identificación de ideas principales, ideas específicas, estados de ánimo de los locutores, registros de textos, etc. Por último, el quinto apartado corresponde a la producción oral y está compuesto 
por cuatro tipos de ejercicios que debe desarrollar en interacción con otro candidato en no más de 15 minutos; en esta sección se presentan estímulos visuales para motivar y evaluar la habilidad para interactuar en conversaciones que ocurren en diversos contextos.

Dos meses después de haber rendido el examen, los candidatos reciben un informe de resultados emitido por la Universidad de Cambridge, el cual expresa de manera individual el nivel general de competencia comunicativa en inglés alcanzado por cada estudiante en el test. Este se expresa en letras que corresponden a cinco grados de competencia comunicativa. Como se muestra en la siguiente tabla, cada una de estas letras corresponde a un rango porcentual de logro. A, B y C indican aprobación, D y E señalan reprobación. Estos grados se basan en la consideración de los resultados en general, es decir, aquellos obtenidos en todas las partes del examen.

Tabla 1. Correspondencia entre categorías, grados y rango porcentual de logro de objetivos en FCE.

\begin{tabular}{|c|c|c|}
\hline Categorías & Grados & Rango porcentual de logro \\
\hline Aprobación & A & $80 \%$ y más \\
\hline & B & $75 \%$ a $79 \%$ \\
\hline Reprobación & C & $60 \%$ a $74 \%$ \\
\hline & D & $55 \%$ a $59 \%$ \\
\hline
\end{tabular}

Por otra parte, el informe de resultados especifica también los rendimientos parciales en los cinco apartados del examen mediante una gráfica, los que se muestran según una escala de cuatro categorías: excepcional, bueno, limítrofe, pobre. En dicho gráfico, la coordenada X corresponde a los aspectos evaluados (comprensión auditiva, comprensión de lectura, producción oral, producción escrita, uso del inglés) y la coordenada $\mathrm{Y}$ corresponde a los criterios de logro en cada uno de dichos aspectos.

La figura 1 presenta un informe real de los resultados obtenidos por un sujeto.
Figura 1. Ejemplo de informe de evaluación de competencia en FCE.

\begin{tabular}{|c|c|c|}
\hline Qualification & Grade & Result \\
\hline $\begin{array}{l}\text { FIRST CERTIFICATE IN ENGLISH } \\
\text { Candidate profile }\end{array}$ & C & PASS \\
\hline $\begin{array}{l}\text { Exceptional } \\
\text { Good }\end{array}$ & & Speaking \\
\hline Borderline Reading & Use of English & Listening \\
\hline
\end{tabular}

Con el fin de poder establecer relaciones cuantitativas que permitieran hacer análisis estadísticos, se tradujeron los criterios de logro expresados en el gráfico a términos numéricos, asignando una puntuación basada en centímetros de distancia respecto a un origen " 0 ". De este modo, se generaron indicadores numéricos "uctuantes entre un valor mínimo “0” y un valor máximo “5”. La tabla 2 muestra el resultado de este procedimiento:

Tabla 2. Correspondencia entre puntajes y niveles de logro de competencia comunicativa en FCE.

\begin{tabular}{|c|c|}
\hline Valor & Nivel de logro \\
\hline De 0 a 2 & Pobre \\
\hline De 2,1 a 3 & Limítrofe \\
\hline De 3,1 a 4 & Bueno \\
\hline De 4,1 a 5 & Excepcional \\
\hline
\end{tabular}

- Perfil estratégico de aprendizaje de lenguas extranjeras

Otra fuente de datos considerados en este estudio, corresponde a un perfil de estrategias de aprendizaje de inglés como lengua extranjera que caracteriza al grupo de sujetos que constituyen la muestra de este estudio. Este perfil fue obtenido en un estudio anterior desarrollado por las autoras, mediante la aplicación del SILL, en su versión 7.0, diseñado por Rebecca Oxford especialmente para estudiantes de inglés como segunda lengua o lengua extranjera (Sanhueza y Burdiles, 2011). 
El perfil da cuenta de la frecuencia de uso de las estrategias de memorización, cognitivas, de compensación, metacognitivas, afectivas y sociales.

\section{Técnicas de procesamiento y análisis de la información}

En la primera etapa, se describió la variable competencia comunicativa en inglés, en términos de la distribución porcentual de los sujetos en las categorías aprobados y reprobados en el FCE y cómo se distribuyeron los mismos en cada una de las categorías, según el grado general de aprobación o reprobación: A, B, C y D, E, respectivamente. Luego, se analizó la tendencia general observada en relación al nivel de competencia comunicativa (excepcional, bueno, limítrofe o pobre) demostrada en cada uno de los cinco aspectos evaluados en el FCE (comprensión de lectura, producción escrita, comprensión auditiva, producción oral y uso del idioma). En seguida, los análisis se centraron en la distribución porcentual de sujetos por nivel de competencia comunicativa en cada uno de los aspectos evaluados en el examen.

\section{Resultados y discusión}

En conformidad con el primer objetivo de esta investigación, a saber, describir el nivel de competencia comunicativa en inglés alcanzado por un grupo de escolares chilenos en el examen FCE, a continuación se presentan y comentan los resultados.

\section{Resultados de nivel de competencia comunicativa en inglés}

Los análisis tendientes a describir cuál es el nivel de competencia comunicativa en inglés alcanzado por los sujetos de la muestra en el examen FCE se realizaron, primero, desde una perspectiva general y, luego, desde una perspectiva más específica.

A continuación se presentan los resultados de los análisis estadísticos porcentuales que informan acerca de la distribución de los sujetos, según criterios de aprobación y reprobación, y según los cinco niveles generales de logro de competencia comunicativa establecidos en el informe de resultados del FCE: A, B, C, D y E. Posteriormente, se señalan los resultados de acuerdo con los cuatro niveles de competencia comunicativa: excepcional, bueno, limítrofe y pobre, alcanzados en cada uno de los cinco aspectos evaluados
Diagnóstico de la competencia comunicativa en inglés de un grupo de escolares chilenos: puntos de encuentro con su perfil estratégico

en el examen. Finalmente, se muestran los resultados de la distribución de los sujetos por nivel de logro en cada uno de los aspectos evaluados en el examen FCE.

En primer término, exponemos en la figura 2 los resultados de la distribución de los sujetos en las categorías aprobados y reprobados, como también en los cinco niveles generales de logro de competencia comunicativa.

Figura 2. Distribución Aprobados/Reprobados según nivel de logro en FCE.

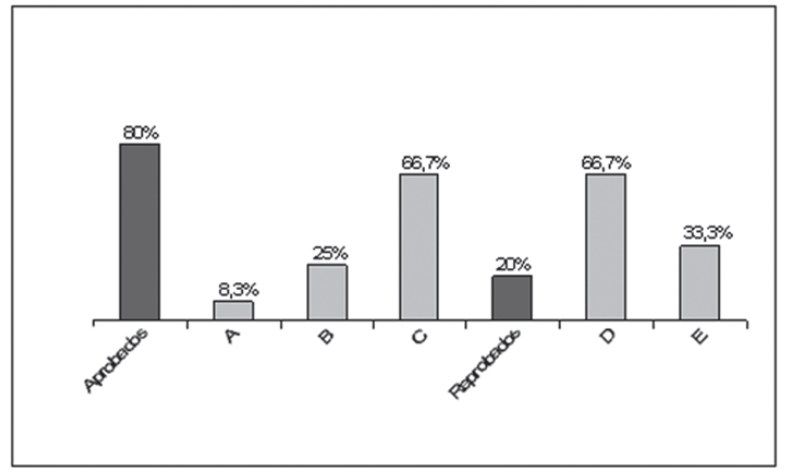

Como se puede apreciar a partir de la figura 2, el porcentaje de alumnos que aprobó el FCE corresponde a la mayoría de los sujetos de la muestra. Además, se observa que la mayor parte de los aprobados $(66,7 \%)$ logró un nivel de competencia $\mathrm{C}$, es decir, un nivel de aprobación mínimo que en el examen se ubica en el rango $60 \%$ a $74 \%$ de logro. En relación con los reprobados, la mayoría $(66,7 \%)$ se ubicó en el nivel $\mathrm{D}$ de competencia, nivel que el examen ubica entre el $55 \%$ a $59 \%$ de logro.

Los resultados informan que, en términos generales, el nivel de competencia comunicativa demostrado por la mayoría de los sujetos de la muestra (80\%) en el examen FCE corresponde, de acuerdo a la Universidad de Cambridge, a un nivel intermedio-alto de competencia en inglés. Estos resultados parecen halagüeños; no obstante, en la comunidad educativa de este grupo de estudiantes las expectativas previas a la medición del FCE eran superiores, dadas las características académicas de los sujetos, ya señaladas al describir la muestra.

Ahora bien, los cálculos de medidas de tendencia central, hechos en relación con el nivel de competencia comunicativa demostrado por los estudiantes en cada uno de los cinco aspectos evaluados en el FCE, condujeron a los resultados que se exponen a continuación: 
Figura 3. Nivel General de Logro por Aspecto Evaluado en FCE.

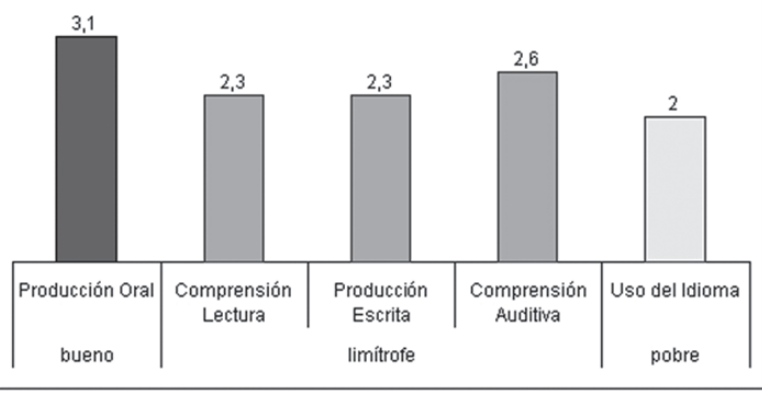

Como muestra la figura 3, en ninguno de los aspectos se obtuvo un nivel de competencia superior a 4,1, es decir, a excepcional (cf. tabla 2). El nivel de logro más alto (bueno) se observó en la habilidad de producción oral; el nivel de logro más bajo (pobre) se observó en el aspecto uso del idioma. En relación al resto de las habilidades, el nivel de logro fue limítrofe, destacando levemente aquel en referencia a comprensión auditiva.

En tercer término, presentamos los resultados de la distribución de los sujetos por nivel de logro en cada uno de los aspectos evaluados en el examen FCE; vale decir: comprensión de lectura, producción escrita, comprensión auditiva, producción oral y uso del idioma.

Figura 4. Distribución según Nivel de Logro por Aspecto FCE.

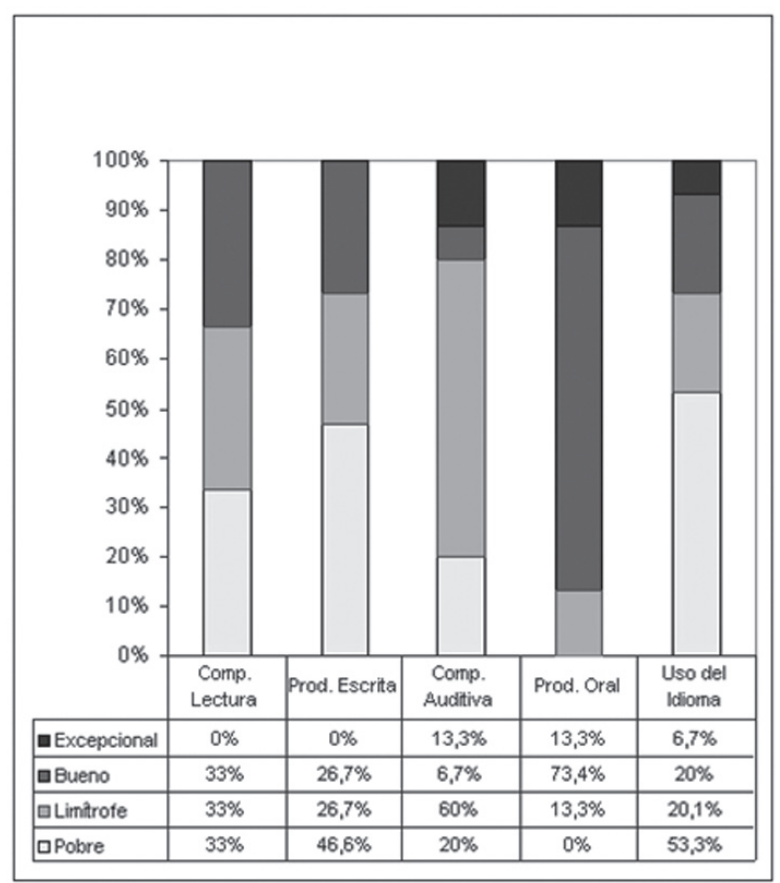

La información graficada en la figura 4 muestra una distribución homogénea de los sujetos según su competencia en comprensión lectora entre las categorías bueno, limítrofe y pobre. En cuanto a producción escrita, se evidencia que la mayoría de ellos se ubicó en la categoría pobre y el resto se distribuyó homogéneamente en las categorías limítrofe y bueno. Tanto en comprensión de lectura como en producción escrita, no hubo sujetos en la categoría excepcional. Respecto a comprensión auditiva, cabe destacar que más de la mitad de los sujetos se ubicó en la categoría limítrofe y que, unidos a los ubicados en la categoría pobre, constituyen un $80 \%$ de la muestra. Con relación a producción oral, un porcentaje significativamente mayor $(86,7 \%)$ se ubicó en el tramo de las categorías bueno y excepcional. Es llamativo también que el porcentaje de sujetos ubicado en la categoría limítrofe es significativamente menor que en los otros aspectos evaluados por el FCE, y que ningún sujeto se ubicó en la categoría pobre. Por último, fue posible advertir que en uso del idioma, dos tercios de los sujetos se ubicaron en los niveles pobre y limítrofe.

En síntesis, en producción escrita y uso del idioma, la tendencia corresponde a la categoría pobre; en comprensión auditiva, corresponde a la categoría limítrofe. Solo en el aspecto producción oral la tendencia corresponde a la categoría bueno.

La tendencia a un nivel pobre de competencia en producción escrita podría relacionarse con el bajo nivel obtenido en uso del idioma, donde se evidencia un disminuido conocimiento del sistema de la lengua. Un buen escritor requiere tener conocimiento y manejo de un vocabulario amplio y de estructuras gramaticales de mayor complejidad. Por otra parte, un buen escritor debe desarrollar y usar un conjunto de estrategias de escritura tales como estar muy consciente de la audiencia para quien escribe, planificar, revisar y ajustar su escritura, recurriendo al parafraseo y sinónimos. Todo lo anterior se torna más complejo cuando se trata de componer un producto escrito enmarcado en tiempos restringidos, lo cual conlleva una carga afectiva adicional. Es posible que estos resultados sean indicio de que el desarrollo de la competencia en producción escrita 
esté limitado por la escasa oferta de oportunidades para componer por escrito en las clases de lengua extranjera y en contextos funcionales.

Los resultados expuestos en la figura 4 indican que más de la mitad de los sujetos obtuvieron niveles limítrofe y pobre en comprensión lectora (un 66\%) y en comprensión auditiva (un 80\%). Desde un punto de vista, la modalidad oral y la escrita pueden ser consideradas como normas autónomas de lengua, puesto que responden a necesidades culturales específicas, se asocian con prácticas sociales particulares y están relacionadas con ciertas expresiones de significado estructural. Sin embargo, y dado que esta mirada constituye una simplificación del fenómeno comunicativo, hoy se propone la idea del continuum, según la cual los fenómenos se dan dentro de un espectro y solo se diferencian en grados o matices (Mostacero, 2004). En conformidad con esta idea, entonces, la competencia comunicativa se desarrolla contextualizada en un conjunto de sistemas que van de la oralidad a la escritura y viceversa. Ahora bien, tanto en lo referido a la comprensión lectora como a la oral, debe entenderse que la comprensión es un macroproceso cognitivo, compuesto de microprocesos de diversos grados de complejidad (Parodi, 2003), que requiere desde la intervención de nuestros sistemas sensoriales (estímulos acústicos y estímulos visuales) hasta el desencadenamiento de complejos procesos inferenciales.

El desempeño poco exitoso en estos dos aspectos, aparentemente diferentes, de la evaluación FCE revela de manera coincidente la existencia de problemas generales de comprensión de discursos literarios y no literarios en dos modalidades de expresión: los registros orales y los escritos. En ambos casos, el examen FCE proporciona al evaluado la oportunidad de demostrar la asignación de significado a partir de algunos textos auténticos como libros, cartas, diarios, revistas, propaganda, entrevistas, discusiones, charlas y conversaciones. En relación a estas tareas de comprensión, los aspectos que se manifiestan en niveles deficitarios o pobres en los sujetos de la muestra, tienen relación con la determinación de ideas generales y específicas, con la identificación de estructuras textuales y el esta- blecimiento de inferencias (University of Cambridge ESOL Examinations).

Por otra parte, el buen nivel de competencia comunicativa demostrado por los estudiantes en producción oral puede explicarse porque los sujetos comparten la experiencia de haber estado expuestos, durante todos sus años de colegio, a situaciones naturales de interacción oral en inglés, lo que potenció en ellos un alto grado de seguridad asociada al uso comunicativo de la lengua extranjera. Así mismo, en el aspecto producción oral, los sujetos recurren a elementos paralingüísticos para apoyar la producción y la comprensión, lo que les permite negociar significados en el momento en que están siendo sometidos a la evaluación. Cabe destacar que la modalidad de evaluación de los otros aspectos del FCE se enmarca en un espectro de respuestas definidas a priori y, por lo mismo, más restringido.

Ahora bien, el bajo nivel de competencia comunicativa demostrado en uso del idioma, el cual puede significar carencias en aspectos gramaticales, pudiera obedecer a que, si bien la institución de donde se extrajo la muestra declara en sus planes y programas la enseñanza del inglés bajo un enfoque comunicativo, la interpretación que se ha hecho de este ha enfatizado la fluidez en desmedro de la precisión en el uso del idioma. Esta interpretación, como lo expresa Medina (1998), ha sido históricamente un tema de discusión en el área de la didáctica de lenguas extranjeras, y podría evitarse si se adopta lo que él ha denominado una corriente "sistémicocomunicativa”. Este enfoque integra los conceptos de precisión y fluidez con el fin de lograr una unidad en la forma, el significado y la función, y se aproxima al concepto de competencia comunicativa que subyace al modelo teórico de Canale y Swain (1980), el cual describe la competencia comunicativa en base a cuatro áreas fundamentales: competencia gramatical, competencia sociolingüística, competencia discursiva y competencia estratégica.

Como es posible ver, privilegiar la fluidez sobre la precisión en el proceso de enseñanza de un idioma extranjero no garantiza un alto nivel de competencia comunicativa en aspectos como producción escrita y uso del idioma, en los cuales se requiere de un alto 
dominio de competencia gramatical, entendida esta como el "conocimiento y uso correcto de las reglas gramaticales, del léxico, de la morfología, la sintaxis y la fonología en la lengua extranjera" (Canale y Swain, 1980).

Hasta ahora hemos dado cuenta de la competencia comunicativa demostrada por los sujetos de la muestra en el examen internacional FCE. En lo que sigue, revisitaremos estos resultados a la luz de un perfil estratégico que caracteriza a este mismo grupo de sujetos.

\section{Puntos de encuentro entre nivel de competencia comunicativa y uso de estrategias de aprendizaje de inglés}

A continuación, se hace referencia a algunos de los resultados de un estudio realizado anteriormente por Sanhueza y Burdiles (2011), cuyo objetivo fue establecer la caracterización de un perfil de estrategias de aprendizaje de inglés como lengua extranjera. En dicho estudio se utilizó el SILL (Oxford, 1990) para evaluar el uso de estrategias de aprendizaje que caracterizaba al mismo grupo de estudiantes que conforma la muestra de la presente investigación.

Ahora bien, en los resultados expuestos en la figura 4, se observó que el grupo en estudio alcanza destacados niveles de competencia en producción oral. Además de las explicaciones ya adelantadas, creemos que este nivel de proficiencia podría responder también a las estrategias de aprendizaje que caracterizan a los 15 sujetos de la muestra, las que están señaladas en la figura 5:

Figura 5. Frecuencia de Uso Estrategias de Aprendizaje de Inglés como Idioma Extranjero.

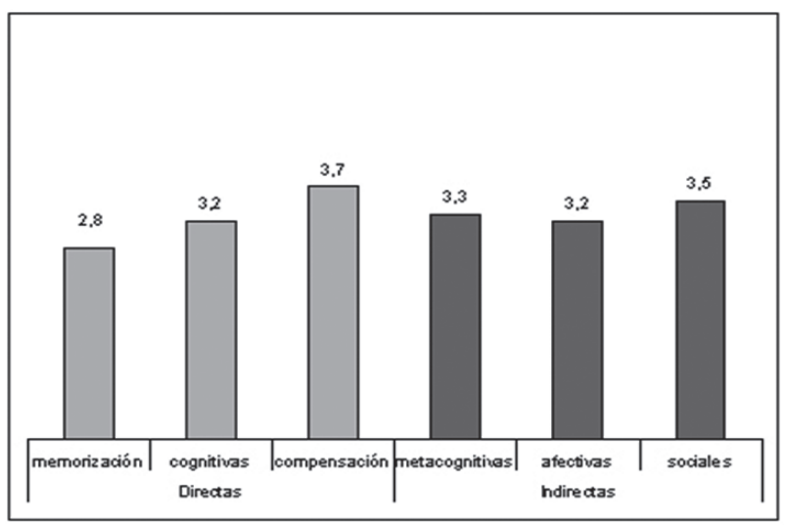

Según los resultados expuestos en la figura 5, el grupo tiende a usar con cierta regularidad todas las estrategias, aunque las más usadas son las de compensación y las sociales. En el otro extremo, se destacan las estrategias de memorización como las menos usadas. Esta misma tendencia se repite si el análisis se realiza considerando solo a los 12 sujetos que aprobaron el FCE.

A nuestro entender, las tendencias mencionadas guardan relación con los altos niveles de competencia oral demostrada en el FCE, puesto que las estrategias de compensación y las sociales corresponden a aquellas que contribuyen en mayor medida a desarrollar mejores niveles de fluidez. El uso de estrategias de compensación tiene relación con la necesidad de vencer las limitaciones lingüísticas enfrentadas por los estudiantes al momento de usar la lengua con fines comunicativos, dado que estas estrategias lo impulsan a mantenerse enfocado en la tarea y evitan que deje de lado la práctica de lengua que esta implica. Las estrategias sociales, en tanto, tienen relación con el aprendizaje cooperativo, con la capacidad de interactuar con otros en beneficio del logro de mejores aprendizajes. A este respecto, podemos sostener que los estudiantes exitosos se caracterizan por actuar con mayor confianza en una segunda lengua y asumen la interacción con otros como una instancia de mejoramiento de la competencia comunicativa en esa lengua (Gregersen et al, 2001; Sanhueza y Burdiles, 2011).

Un interesante ejemplo de la relación entre competencia oral y uso de estrategias, nos lo proporciona el caso del sujeto 14 quien, aunque aprobó el FCE con un nivel de competencia limítrofe (C), y alcanzó -como lo indica la figura 6- niveles pobres y limítrofes en los diferentes aspectos, destaca en producción oral con un nivel excepcional $(4,1)$.

Figura 6. Informe FCE Sujeto 14.

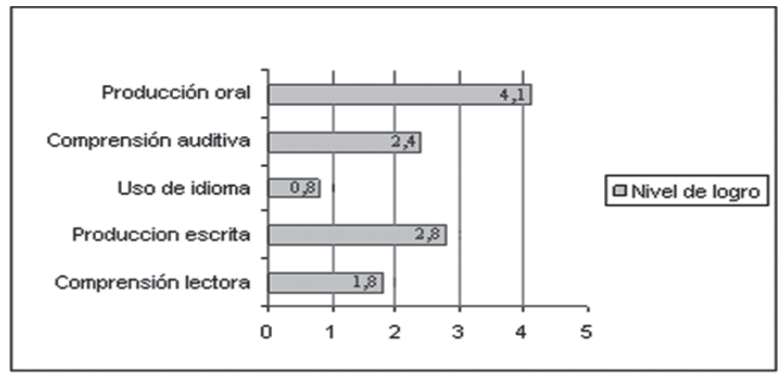


Pues bien, este mismo sujeto, como lo indica la figura 7 , obtuvo altos niveles de frecuencia de uso en las estrategias de compensación $(3,7)$.

Figura 7. Informe SILL Sujeto 14.

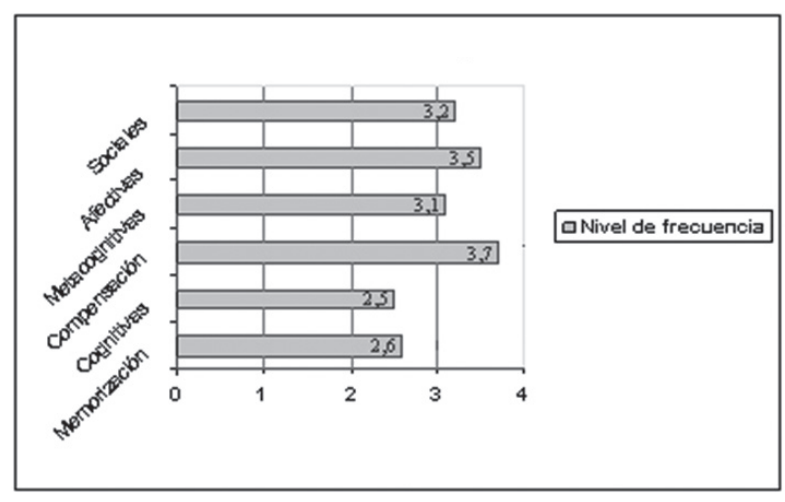

Las estrategias de compensación pudieran haberle permitido subsanar el déficit lingüístico que revelan sus otros resultados y, a pesar de ello, destacarse en su desempeño conversacional. Es llamativo, también, el nivel alto de uso de estrategias afectivas puesto que, como lo indica la teoría, estas estrategias que Oxford (1990) categoriza como indirectas, inciden en el manejo global del aprendizaje, ya que regulan las emociones que un usuario de lengua extranjera pudiera experimentar al momento de producir mensajes orales en LE.

Otro de los resultados generales observados sobre estrategias en este grupo de sujetos, es que las menos utilizadas fueron las de memorización (cf.: figura 5). Estas estrategias inciden en potenciar lo que Canale y Swain (1980) denominan competencia gramatical (o lingüística), relacionada con el conocimiento y uso correcto de reglas (léxicas, morfológicas, sintácticas, fonológicas) de la lengua extranjera. El uso poco frecuente de estrategias de memorización también se podría asociar al bajo nivel de competencia demostrado por los sujetos en el FCE, en los aspectos de uso del idioma y producción escrita. Téngase presente que el correcto uso de vocabulario y estructuras gramaticales son los elementos que constituyen el énfasis puesto en la evaluación de la precisión en lo que respecta a las tareas de uso del idioma y producción escrita contenidas en este examen.
Diagnóstico de la competencia comunicativa en inglés de un grupo de escolares chilenos: puntos de encuentro con su perfil estratégico

Si bien el conocimiento léxico y de estructuras gramaticales es esencial en las primeras etapas de aprendizaje de un idioma extranjero (Oxford, 1990; Celce-Murcia, 2001; Brown, 2001), lo esperable es, tal como se observa en el caso que a continuación se reporta, que estudiantes expertos no recurran preferentemente a este tipo de estrategia. Esta fue precisamente la situación observada con el sujeto 1, quien aprobó el FCE con A, es decir, con un $80 \%$ o más de logro.

Figura 8. Informe FCE Sujeto 1.

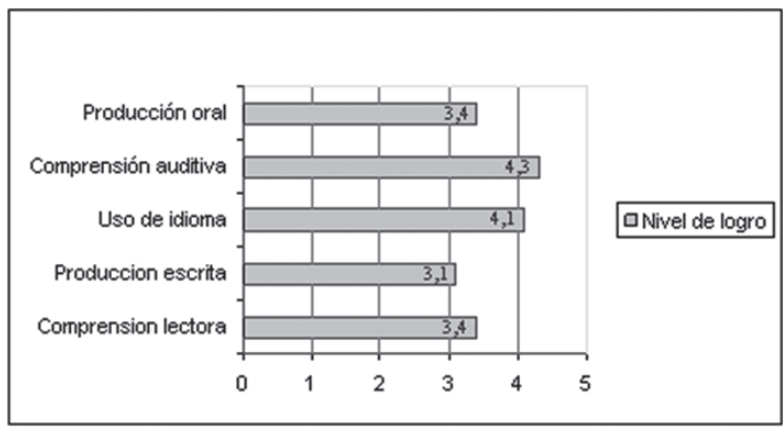

Como se puede ver en la figura 8 , este sujeto se caracteriza porque sus niveles de logro por aspecto evaluado en el FCE oscilan entre bueno y excepcional. Además, como lo indica la figura 9 , se caracteriza por alcanzar puntajes medios en el uso de varias estrategias; sin embargo, llama la atención que en las estrategias de memorización tenga puntaje calificado de bajo $(1,8)$.

Figura 9. Informe SILL Sujeto 1.

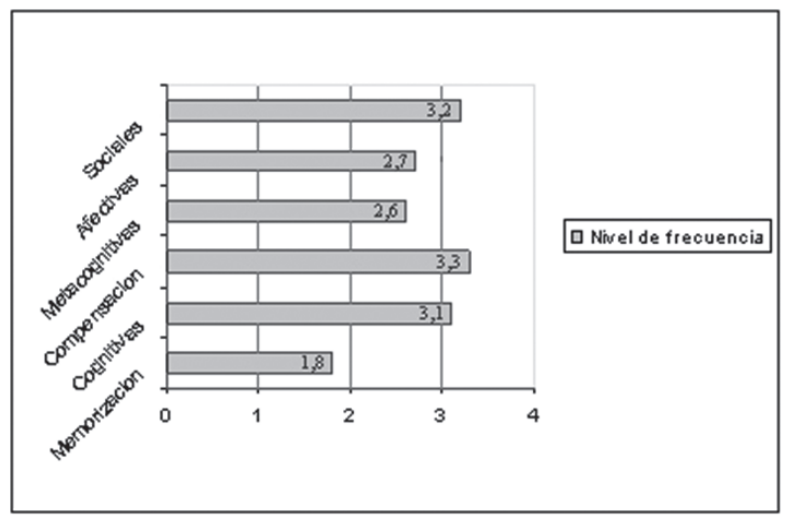

A la luz de estos datos, podríamos afirmar que el sujeto 1 es competente desde una perspectiva comunicativa y que, desde una perspectiva estratégica, ha soslayado el uso de estrategias de memorización. 
Dicho de otro modo, sostenemos que, a medida que se avanza desde el aprendizaje a la adquisición de una lengua, el uso del idioma deviene en un proceso menos consciente acerca de cómo es la lengua y más eficiente en su uso.

\section{Conclusiones}

El presente estudio tuvo como propósito principal describir el nivel de competencia comunicativa alcanzado en el FCE, por un grupo de estudiantes chilenos de educación media. Paralelamente, se profundizó este diagnóstico mediante el establecimiento de puntos de encuentro entre la descripción de la competencia comunicativa en inglés de dichos sujetos y el perfil de estrategias de aprendizaje de lengua extranjera que caracteriza a los mismos. El análisis de los resultados nos conduce a las conclusiones que a continuación se señalan.

El grupo de estudiantes que constituye la muestra se caracteriza mayoritariamente por manifestar un nivel intermedio-alto de competencia comunicativa en inglés, certificado por el examen FCE, en conformidad con los estándares establecidos por el Marco común europeo. En este sentido, aun cuando la tendencia del grupo fue aprobar con puntajes generales mínimos de aprobación, se puede afirmar que su establecimiento educacional de origen podría dar por cumplidos los objetivos perseguidos en el desarrollo de la competencia en inglés.

Del desempeño demostrado, los resultados más destacables son los relacionados con la competencia en producción oral. Esto quiere decir que los sujetos manifiestan gran habilidad para interactuar eficientemente en conversaciones referidas a temas variados; sin embargo, revelan debilidades en otros aspectos comunicativos. El buen nivel de competencia comunicativa demostrado en producción oral puede explicarse, fundamentalmente, por el destacado uso que hacen de estrategias de compensación, que contribuyen a superar eventuales limitaciones lingüísticas al momento de usar la lengua con fines comunicativos, dado que impulsan al hablante a mantenerse enfocado en la tarea y a hacer uso eficiente de elementos paraverbales. Otro factor que, en este caso, se relaciona con el exitoso desempeño oral, es el alto uso de estrategias sociales que el grupo reporta, estrategias caracterizadas por favorecer la fluidez, en la medida en que se estimula cooperativamente la interacción comunicativa.

Por otro lado, el grupo de estudiantes observado en este estudio se caracteriza por manifestar niveles deficientes en varios otros aspectos de la competencia comunicativa, tales como la comprensión lectora y auditiva, la producción escrita y el uso del idioma.

El bajo nivel de competencia comunicativa demostrado en uso del idioma puede significar carencias en aspectos gramaticales. A su vez, la tendencia a un nivel pobre de competencia en producción escrita guarda relación con deficiencias en uso del idioma, donde se evidencia un disminuido conocimiento del sistema de la lengua. Los problemas de comprensión, en tanto, se manifiestan igualmente en relación con los registros orales y los escritos.

Este panorama poco alentador, que contrasta con la alta fluidez demostrada en la competencia oral, podría explicarse por el escaso dominio de la precisión en aspectos formales de la lengua. A este respecto, no deja de llamar la atención que las estrategias menos frecuentes en este grupo de sujetos fueron las de memorización, estrategias que inciden en potenciar la competencia gramatical o lingüística (Canale y Swain, 1980), relacionada con el conocimiento y uso correcto de reglas (léxicas, morfológicas, sintácticas, fonológicas) de la lengua extranjera.

Lo anterior sugiere que, si bien la enseñanza del idioma inglés en el colegio bilingüe del que proviene la muestra se ha abordado desde un enfoque comunicativo, el énfasis ha sido el desarrollo de la fluidez en desmedro de la precisión. Esta situación puede constituir un obstáculo para el logro del conjunto de competencias que configuran la competencia comunicativa, a saber: la competencia gramatical (o lingüística), discursiva, sociolingüística, estratégica, sociocultural, de aprendizaje, cognitiva, afectiva, comportamental e intercultural (Medina, 1998).

A partir del diagnóstico que se desprende de estudios como el que en este artículo hemos descrito, sería posible pensar en ajustes susceptibles de implementar en los procesos de enseñanza y 
aprendizaje de la asignatura de inglés en el contexto escolar. Considerando las observaciones hechas en esta descripción del nivel de competencia comunicativa alcanzado por un grupo de estudiantes, y su relación con el uso de estrategias de aprendizaje de lengua extranjera, a continuación planteamos algunas sugerencias que podrían contribuir a desarrollar mejores niveles del conjunto de competencias necesarias para ser un usuario competente del inglés.

1. Seleccionar, o diseñar, herramientas evaluativas diagnósticas que pudieran implementarse en distintos momentos del proceso de enseñanza y aprendizaje del inglés a nivel escolar.

2. Revalorizar el rol de la enseñanza formal de la gramática y el vocabulario bajo el enfoque comunicativo de enseñanza del inglés, con perspectivas a integrar el desarrollo de la precisión y la fluidez al momento de usar el idioma.

3. Incluir la enseñanza directa, informativa y explicativa del conjunto de estrategias de aprendizaje, no solo de aquellas que contribuyen en mayor medida al desarrollo de la fluidez, como las estrategias de compensación, las sociales y las afectivas, sino también, y con especial énfasis, las que se constituyen como imprescindibles para alcanzar un buen nivel de precisión en el uso del idioma.

4. Enfatizar la enseñanza y el uso de las estrategias de memorización que se han evidenciado como las más débiles y que, dada la importancia que estas cobran al momento de almacenar y recobrar vocabulario y estructuras gramaticales, interfieren en el logro de un buen nivel de competencia comunicativa en producción escrita y uso preciso del idioma.

5. Aumentar la oferta de oportunidades que apunten al desarrollo de la competencia en producción escrita en contextos funcionales.

6. Promover el uso de un conjunto de estrategias de escritura, tales como estar muy consciente de la audiencia para quien escribe, planificar, revisar y ajustar su escritura, recurriendo al parafraseo y sinónimos, con el fin de respon-
Diagnóstico de la competencia comunicativa en inglés de un grupo de escolares chilenos: puntos de encuentro con su perfil estratégico

der a los requerimientos de una comunicación escrita eficiente.

7. En suma, este estudio cobra importancia en el ámbito de la investigación de las potenciales relaciones entre la competencia comunicativa y las estrategias de aprendizaje específicas. Consideramos que la evaluación de ambos factores puede y debe ser considerada antes del diseño, implementación y evaluación de intervenciones tendientes a optimizar los procesos de enseñanza-aprendizaje del inglés como idioma extranjero en los colegios.

\section{Referencias}

Allwright, R. (1981). What Do We Want Teaching Materials For? In ELT Journal, vol. 36(1), 5-18.

Bialystok, E. (1981). The Role of Conscious Strategies in Second Language Proficiency. In The Modern Language Journal, vol.65(1), 24-35.

Brown, D. (2001). Teaching by Principles: An Interactive Approach to Language Pedagogy. Nueva York: Addison Wesley Longman.

Canale, M. (1983). From Communicative Competence to Communicative Pedagogy. In J. Richards y R. Schmidt (Eds.), Language and communication. Londres: Longman.

Canale, M. \& Swain, M. (1980). Theoretical Bases of Communicative Approaches to Second Language Teaching and Testing. In Applied Linguistics, 1, 1-47.

Cartes, N., Solar, M. y Quintana, R. (2004). Aula inteligente y aula tradicional: un estudio experimental. En Estudios de Lingüística Aplicada, 37(21), 137-161. UNAM.

Casar, L. y Hernández, A. (2000). La aplicación de las estrategias de aprendizaje en la enseñanza del inglés. Documento en línea. Recuperado en abril de 2011. Disponible en http://www.nuestraldea.com/aprendizaje_ingles.html

Celce-Murcia, M. (Ed.). (2001). Teaching English as a Second or Foreign Language. Boston: Heinle Cengage Learning.

Chamot, A. (2004). Issues in Language Learning Strategy Research and Teaching. In Electronic Journal of Foreign Language Teaching, vol.1(1), 14-26. 
Chamot, A. et al. (1987). A Study of Learning Strategies in Foreign Language Instruction: First Year Report. Washington D.C: Interamerica Research Associates.

Chomsky, N. (1965). Aspects of the Theory of Syntax. Cambridge, Mass: The MIT Press.

Cohen, A. \& Hosenfeld, C. (1981). Some Uses of Mentalistic Data in Second Language Research. In Language Learning: A Journal of Research in Language Studies, vol.31(2), 285-313.

Cohen, A. (1990). Language Learning: Insights for Learners, Teachers and Researchers. Boston: Heinle \& Heinle.

Cohen, D., Weaver, S. \& Tao-Yuan, L. (1996). The Impact of Strategies-Based Instruction on Speaking a Foreign Language. Documento en línea. Recuperado en abril de 2011. Disponible en http://www.carla.umn.edu/ about/profiles/CohenPapers/SBIimpact.pdf

Dansereau, D. et al. (1975). The Development of Learning Strategies Curriculum. In J.R. O'Neil (ed.). (1978), Learning Strategies. Educational technology series. Nueva York, San Francisco, Londres: Academic Press.

Doddis, A., Novoa, P. y Tabilo, X. (2003). Estrategias léxicas de comunicación en la producción oral de aprendientes de inglés como segunda lengua: un estudio longitudinal. En Revista Lenguas Modernas, ( $\left.\mathrm{N}^{\circ} 28-29\right), 213-228$.

Ellis, R. (1994). The Study of Second Language Acquisition. Oxford: Oxford University Press.

Ellis, R. (2003). Understanding Second Language Acquisition. Oxford: Oxford University Press.

Germany, P. y Cartes, N. (2000). Léxico disponible en inglés como segunda lengua en instrucción formalizada. En Estudios Pedagógicos, ( $\left.{ }^{\circ} 26\right)$, 39-50.

Gregersen, T., Vera, R., Pino, P. \& Espinoza, L. (2001). Can Foreign Language Learning Strategies Turn Into Crutches? A Pilot Study on the Use of Strategies by Successful and Unsuccessful Language Learners. En Revista Signos, (N³4), 49-50, 101-111.

Griffiths, C. \& Parr, J. (2000). Language Learning Strategies, Nationality, Independence and Proficiency. In Independence, ( $\left.\mathrm{N}^{\circ} 28\right), 7-10$.

Halliday, M. (1970). Language Structure and Language Function. In J. Lyons (Ed.), New Horizons in Linguistics (pp. 140-165). Harmondsworth: Penguin Books Ltd.
Hymes, D. (1972). On Communicative Competence. In J.B. Pride \& D. Hymes (Eds.), Sociolinguistics. Harmondsworth: Penguin Books Ltd.

Hosenfeld, C., Arnold, V., Kirchofer, J., Laciura, J. \& Wilson, L. (1981). Second Language Reading: A Curricular Sequence for Teaching Reading Strategies. In Foreign Language Annals, vol.14(5), 415-422.

Instituto Cervantes. (2002). Marco de referencia europeo para el aprendizaje, la enseñanza y la evaluación de lenguas. Madrid. Documento en línea. Recuperado en abril de 2011. Disponible en http://cvc.cervantes. es/obref/marco

Lessard-Clouston, M. (1997). Language Learning Strategies: An Overview for L2 Teachers. In M. Hismanoglu, (2000), The Internet TESL Journal, vol. 6(8). Documento en línea. Recuperado en abril de 2011. Disponible en http://iteslj.org/Articles/HismanogluStrategies.html

Lomas, C. y Osoro, A. (Comps.). (1994). El enfoque comunicativo de la enseñanza de la lengua. Barcelona: Ediciones Paidós Ibérica, S.A.

McGroarty, M. (1987). Patterns of Persistent Second-Language Learners: Elementary Spanish. Paper presentado en el congreso anual de TESOL. Miami, Florida.

Medina, A. (1998). La competencia comunicativa en lenguas extranjeras desde una perspectiva configuracional. Documento en línea. Recuperado en abril de 2011. Disponible en http://www.monografias.com/ trabajos19/competencia-comunicativa/competencia comunicativa.shtml

Monereo, C. (Coord.). (2004). Estrategias de enseñanza y aprendizaje. Formación del profesorado y aplicación en la escuela. Barcelona: Editorial Graó.

Mostacero, R. (2004). Oralidad, escritura y escrituralidad. En Sapiens, vol.5(1), 53-75.

Nyikos, M. \& Oxford, R. (1993). A Factor Analytic Study of Language Learning Strategy Use: Interpretations from Information-Processing Theory and Social Psychology. In The Modern Language Journal, vol.77(1), 11-22.

Olivares-Cuhat, G. (2002). Learning Strategies and Achievement in the Spanish Writing Classroom: A Case Study. In Foreign Language Annals, vol.35(5), 561-570.

Oliveras, A. (2000). Hacia la competencia intercultural en el aprendizaje de una lengua extranjera. Madrid: Edinumen. 
O'Malley, J., Chamot, A., Stewner-Manzanares, G., Russo, R. \& Kupper, L. (1985). Learning Strategy Applications with Students of English as a Second Language. In TESOL Quarterly, vol. 19(3), 557-584.

O'Malley, J. \& Chamot, A. (1987). The Cognitive Academic Language Learning Approach: A Bridge To The Mainstream. In TESOL Quarterly, vol 21(2), 227-249.

Oxford, R. (1990). Language Learning Strategies: What Every Teacher Should Know. Boston, MA: Heinle \& Heinle.

Oxford, R. \& Burry-Stock, J. (1995). Assessing the Use of Language Learning Strategies Worldwide with the ESL/EFL Version of the Strategy Inventory for Language Learning (SILL). In System, vol.23(1), 1-23.

Oxford, R. (1996). Language Learning Strategies Around the World: Cross Cultural Perspectives. Hawai: University of Hawaii at Manoa.

Parodi, G. (2003). Relaciones entre lectura y escritura: una perspectiva cognitiva-discursiva. Valparaíso, Chile: Ediciones Universitarias de Valparaíso.

Politzer, R. (1983). An Explanatory Study of Self-Reported Language Learning Behaviours and Their Relation to Achievement. In Studies in Second Language Acquisition, vol. 6, 54-68.

Politzer, R. \& McGroarty, M (1985). An Explanatory Study of Learning Behaviours and Their Relationships to Gains in Linguistics and Communicative Competence. In TESOL Quarterly, vol. 19, 103-123.

Pulido, A. y Pérez, V. (2004). Hacia un concepto de competencia comunicativa integral: un novedoso acercamiento a sus dimensiones. Documento en línea. Recuperado en abril de 2011. Disponible en http:// www.monografias.com/trabajos19/competenciacomunicativa/competencia-comunicativa.shtml

Richards, J. \& Lockhart, C. (1994). Reflective Teaching in Second Language Classrooms. USA: Cambridge University Press.

Rosas, M. (1997). Persistencia y cambio en la enseñanza y aprendizaje del inglés. En Literatura y Lingüística, vol.10, 205- 219.

Rubin, J. (1975). What the "Good Language Learner" Can Teach Us. In TESOL Quarterly, vol. 9, 41-51.

Rubin, J. (1981). Study of Cognitive Processes in SecondLanguage Learning. In Applied Linguistics, vol. 2(11), 118-131.

Sanhueza, G. y Burdiles, G. (2011). Estableciendo un perfil de estrategias de aprendizaje de inglés como lengua
Diagnóstico de la competencia comunicativa en inglés de un grupo de escolares chilenos: puntos de encuentro con su perfil estratégico

extranjera en escolares chilenos. En La enseñanza y aprendizaje del inglés como lengua extranjera. Aproximaciones desde la investigación. RIL, Santiago, Chile.

Stern, H. (1992). Issues and Options in Language Teaching. Oxford: Oxford University Press.

Sutter, W. (1989). Strategies and Styles. Aalborg. Documento en línea. Recuperado en abril de 2011. Disponible en http://www.cal.org/resources/digest/ oxford01.html

Tarone, E. (1983). Some Thoughts on the Notion of "Communication Strategy". In C. Faerch \& G. Kasper (Eds.), Strategies in Interlanguage Communication (pp. 61-74). Londres: Longman.

University of Cambridge ESOL Examinations. Documento en línea. Recuperado en abril de 2011. Disponible en http://www.cambridgeesol.org/exams/index.html

Van Ek, J. (1984). Across the Threshold Readings from the Modern Languages Projects of the Council of Europe. Oxford: Pergamon Press.

Wenden, A. (1986). What Do Second-Language Learners Know About Their Language Learning? A Second Look at Retrospective Accounts. In Applied Linguistics, Vol. 2(7), 186-205.

Wenden, A. y Rubin, J. (Eds.). (1987). Learner Strategies in Language Learning. United Kingdom: Prentice Hall.

Wharton, G. (2000). Language Learning Strategy Use of Bilingual Foreign Language Learners in Singapore. In Language Learning, vol. 50(2), 203-244.

Widdowson, H. (1978). Teaching Language as Communication. Oxford: Oxford University Press. 